\title{
Efficacy and safety of acupuncture in children: an overview of systematic reviews
}

\author{
Chunsong Yang ${ }^{1}$, Zilong Hao², Ling-Li Zhang' and Qin Guo ${ }^{3}$
}

In recent years, acupuncture has increasingly being integrated into pediatric health care. It was used on $\sim 150,000$ children $(0.2 \%)$. We aim to update the evidence for the efficacy and safety of acupuncture for children and evaluate the methodological qualities of these studies to improve future research in this area. We included 24 systematic reviews, comprising 142 randomized controlled trials (RCTs) with 12,787 participants. Only 25\% (6/24) reviews were considered to be high quality (10.00 \pm 0.63$)$. High-quality systematic reviews and Cochrane systematic reviews tend to yield neutral or negative results $(P=$ 0.052, 0.009 respectively). The efficacy of acupuncture for five diseases (Cerebral Palsy (CP), nocturnal enuresis, tic disorders, amblyopia, and pain reduction) is promising. It was unclear for hypoxic ischemic encephalopathy, attention deficit hyperactivity disorder, mumps, autism spectrum disorder (ASD), asthma, nausea/vomiting, and myopia. Acupuncture is not effective for epilepsy. Only six reviews reported adverse events (AEs) and no fatal side effects were reported. The efficacy of acupuncture for some diseases is promising and there have been no fatal side effects reported. Further high-quality studies are justified, with five diseases in particular as research priorities.

A cupuncture is one of the most popular forms of Traditional Chinese Medicine. It has been used to treat various diseases and relieve symptoms for more than 2,500 $\mathrm{y}$ and is growing in prominence in many parts of the world. Data from the United States suggests that the number of adults who used acupuncture increased from 2 million to 3 million between 2002 and 2007, and it was estimated that $4 \%$ of the United States population and $12 \%$ of the Canadian population had used acupuncture at any time in their lives (1-3). Acupuncture is used for a variety of conditions including stroke (4), anxiety and depression (5), irritable bowel syndrome (6), and pain (7).

In recent years, acupuncture has increasingly being integrated into pediatric health care. It was used on $\sim 150,000$ children $(0.2 \%)$ and provided as a part of services in onethird of pediatric pain centers in the United States $(7,8)$. Acupuncture in children has been widely used for asthma, autism spectrum disorder (ASD), cerebral palsy, and complications of cancer (9-12).

Although the actual mechanisms of acupuncture are still unknown, Traditional Chinese acupuncture theory believes that health is achieved by maintaining an uninterrupted flow of Qi. Qi flows through a network of 14 channels, called "meridians", which run along the surface of the human body. There are nearly 400 acupuncture points (acupoints) on the body surface, which linked through the 14 meridians to various organs or viscera (13). By stimulating various meridian points, acupuncture is thought to correct the imbalance of energy in the body and restore natural internal homeostasis (10,14-16).

Apart from traditional needle acupuncture, a wide variety of acupuncture types have been developed including acupressure (the application of pressure to acupoints with nonpenetrating needles or bands), moxibustion (a technique in which the Chinese herb mugwort or Artemesia vulgaris is burned to apply heat to an acupoint), laser acupuncture (the stimulation of traditional acupoints with low-intensity, nonthermal laser irradiation), electroacupuncture (acupuncture needles inserted at acupoints and then stimulated by a device that generates electric pulses), and auricular acupuncture (targets acupoints on the outer ear using manual stimulation or electroacupuncture) (17-19).

Hunt's study included six systematic reviews of acupuncture for children's conditions, with the search strategy updated in 2009 (20). Another review published in 2008 also evaluated the safety and efficacy of acupuncture for children, but again only included six systematic reviews (21). Although acupuncture originated from China and there are many studies published in China, neither of these reviews included systematic reviews published in China and so may have missed some important information. Recently, several systematic reviews and metaanalysis of acupuncture for different types of childhood diseases have been performed and the results were controversial. Therefore, we aim to update the evidence for the efficacy and safety of acupuncture for children and evaluate the methodological qualities of these studies to improve future research in this area.

\footnotetext{
The first two authors contributed equally to this work.

'Department of Pharmacy, Evidence-based Pharmacy Center, West China Second Hospital, Key Laboratory of Birth Defects and Related Diseases of Women and Children, Sichuan University, Chengdu, China; ${ }^{2}$ Department of Neurology, West China Hospital, Sichuan University, Chengdu, China; ${ }^{3}$ Department of Pediatrics, West China Second Hospital, Key Laboratory of Birth Defects and Related Diseases of Women and Children, Sichuan University, Chengdu, China. Correspondence: Ling-Li Zhang (zhlingli@sina.com)

Received 23 September 2014; accepted 26 January 2015; advance online publication 17 June 2015. doi:10.1038/pr.2015.91
} 
RESULTS

\section{Results of the Search}

Our searches initially identified a total of 828 potentially relevant articles. After the removal of duplicates, screening of titles and abstracts, and reading full texts, 24 systematic reviews were included in this overview (Figure 1). Of them, 18 reviews were published in English and the remaining were Chinese.

\section{The Characteristics of Included Studies}

The overview included 24 systematic reviews, comprising 142 randomized controlled trials (RCTs) (median four RCTs) and 12,787 participants (median 279 participants) (Table 1). Eight of the 24 included reviews (33\%) were Cochrane systematic reviews. The first authors originated from China $(n=16)$, United States America $(n=2)$, United Kingdom $(n=2)$, Australia $(n=2)$, Korea $(n=1)$, and Austria $(n=1)$.

The categories of disease included the nervous system $(n=13$, four studies for cerebral palsy, three studies for nocturnal enuresis, two studies for ASD, one study for tic disorders, one study for hypoxic-ischemic encephalopathy, one study for attention deficit hyperactivity disorder, one study for epilepsy); the digestive system ( $n=4$, four for nausea/vomiting); the respiratory system ( $n=3$, three for asthma); and other disorders ( $n=4$, one for mumps, one for myopia, one for infantile colic and pain reduction, one for amblyopia).

\section{Quality Assessment}

Only six out of the 24 reviews (25\%) were considered to be high quality (10.00 \pm 0.63$), 17$ out of $24(72.8 \%)$ were medium quality $(6.12 \pm 0.86)$, and one review was low quality (AMSTAR score of 4). The average quality of Cochrane systematic reviews $(n=8$, $9.13 \pm 1.73)$ was significantly better than that of non-Cochrane systematic reviews $(n=16,5.94 \pm 1.00)(P=0.000)$. Compared with medium or low-quality systematic reviews, high-quality systematic reviews tended to yield significantly more neutral or negative results $(P=0.052)$. Cochrane systematic reviews

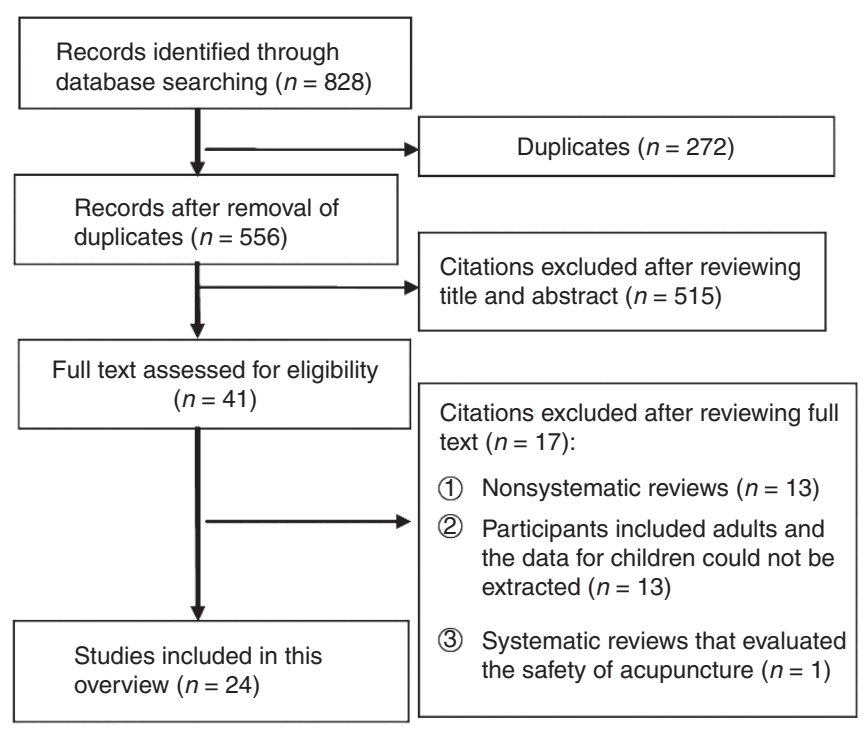

Figure 1. Flow chart of literature screening and selection process. yielded significantly more neutral or negative results than nonCochrane systematic reviews $(P=0.009)$ (Table 2).

Eight out of the 24 systematic reviews (33.3\%) did not register the study protocol before conducting the review. Eighteen out of $24(75 \%)$ did not provide a list of the characteristics of included and excluded studies, with the six exceptions all being Cochrane reviews. Only nine out of 24 reviews (37.5\%) conducted an assessment of publication bias. The status of publication (i.e., gray literature) was used as an inclusion criterion in $20.8 \%$ of reviews $(5 / 24)$ stated and $54.2 \%$ of reviews (13/24) clearly acknowledged the potential sources of support (Table 3).

Non-Cochrane systematic reviews had the following issues: (i) no design protocol; (ii) did not consider publication status; (iii) did not provide a list of included and excluded studies; (iv) no assessment of the publication bias; (v) did not report conflicts of interest (Table 4).

\section{Efficacy and Safety Analysis \\ Nervous System}

Cerebral palsy. Our searches identified four non-Cochrane systematic reviews of cerebral palsy in children, comprising 47 RCTs (4,345 participants). All four reviews compared the efficacy of acupuncture plus conventional treatment with conventional treatment alone (i.e., rehabilitation therapy, drug treatment). The authors all conducted meta-analysis and concluded that acupuncture used as an adjunct to conventional treatment was beneficial for children with cerebral palsy, but further high-quality trials are warranted. Two reviews used the Activities of Daily Living (ADL) scale as an outcome measurement. Zhang et al. (11) found that acupuncture improved ADL in children with cerebral palsy (MD 6.38, 95\% CI 5.15$7.61)$, but the heterogeneity was high $\left(I^{2}=76.7 \%\right)$. Liao et al. (22) also reported improved ADL scores after acupuncture treatment of 3-4 mo (SMD 1.28, 95\% CI 1.00-1.56). Another two reviews used rates of clinical improvements as an outcome measurement; both reviews found acupuncture to be associated with significantly greater improvements than the control group (RR 1.22, 95\% CI 1.11-1.34, OR 3.19, 95\% CI $2.31-4.38)(23,24)$.

Nocturnal enuresis. One Cochrane systematic review and two non-Cochrane systematic reviews comprising 18 RCTs (1,828 participants) focused on nocturnal enuresis, with two of these performing meta-analysis. One review meta-analyzed three RCTs and found a significant difference in the rate of self-defined clinical improvements after treatment of 10-30 d of acupuncture plus Traditional Chinese Medicine compared with Traditional Chinese Medicine alone (OR 5.75, 95\% CI 1.88-17.54) (25). Another review showed that acupuncture was associated with significantly greater improvement in the reduction of the number of wet nights (OR 3.98, 95\% CI 2.2-7.2) (26). Because the outcome measurements and interventions were different in included studies, the Cochrane review did not conduct meta-analysis and concluded that there was weak evidence to support the use of acupuncture for nocturnal enuresis (27). 
Autism spectrum disorder. One Cochrane review and one nonCochrane review assessed the efficacy of acupuncture for ASD, comprising 21 RCTs (888 participants). The Cochrane review showed no difference in primary outcome measurement of core autistic features Ritvo-Freeman Real Life Rating Scale total score between needle acupuncture and sham acupuncture (MD 0.09, 95\% CI -0.03-0.21) (10). The non-Cochrane review did not conduct meta-analysis because of statistical and clinical heterogeneity, and concluded that the efficacy of acupuncture for ASD symptoms was mixed (28).

Tic disorders. One non-Cochrane review evaluated the efficacy of acupuncture for tic disorders, comprising five RCTs (434 participants) (29). The authors meta-analyzed four RCTs and found a significant difference in the rates of clinical improvements between acupuncture and medication (OR 3.39, 95\% CI 1.73-6.65).

Others. Three Cochrane reviews focused on the efficacy of acupuncture for hypoxic-ischemic encephalopathy, attention deficit hyperactivity disorder, and epilepsy respectively, but there were no studies included in two reviews $(30,31)$. Therefore, current evidence did not support acupuncture for treating hypoxic-ischemic encephalopathy and attention deficit hyperactivity disorder. Another review evaluating the efficacy of acupuncture for epilepsy, comprising four RCTs (293 participants), also concluded that the current evidence does not support acupuncture for treating epilepsy (32).

\section{Digestive System}

Nausea/vomiting. Four non-Cochrane reviews examined the efficacy of acupuncture for nausea and/or vomiting, comprising 20 RCTs (1,552 participants). One review determined the efficacy of acustimulation for postoperative nausea and vomiting in children by meta-analyzing 12 RCTs (33). The authors used 24 -h postoperative nausea and vomiting as an outcome measurement and found that all acustimulation modalities reduced vomiting (RR $0.69,95 \%$ CI $0.59-0.80$ ) in 12 trials and reduced nausea (RR $0.59,95 \%$ CI $0.46-0.76$ ) in two trials compared with the control groups.

The second review examined acupuncture effects on prophylaxis of postoperative vomiting in children undergoing tonsillectomy (34). The authors meta-analyzed three RCTs and compared acupuncture plus conventional treatment with conventional treatment alone, and found no significant difference in the number of patients stopping vomiting (OR $0.83,95 \% \mathrm{CI}$ $0.45-1.4)(34)$.

The third review assessed the effects of acupuncture for nausea/vomiting but did not conduct meta-analysis (12). The authors concluded that there was a significant reduction in the need for antiemetics between the treatment group plus acupuncture and the treatment group alone, but there was no significant difference in reported nausea scores (12).

The fourth review examined the efficacy of acupuncture for preventing postoperative nausea and vomiting (35). We extracted the data for children, comprising four RCTs (249 participants). The authors did not perform meta-analysis and concluded that there was no significant benefit from acupuncture compared with placebo or conventional anti-emetics. Therefore, no sufficient evidence showed the effectiveness of acupuncture for nausea/vomiting in children.

\section{Respiratory System}

Asthma. One Cochrane review (36) and two non-Cochrane reviews $(9,17)$ assessed the efficacy of acupuncture for asthma comprising six RCTs (275 participants) with no meta-analysis performed. The authors concluded that no robust evidence exists to support acupuncture as being effective for treating childhood asthma.

\section{Others}

Pain. One non-Cochrane review assessed the safety and efficacy of acupuncture in term and preterm infants and included four RCTs comprising 140 neonates (37). Neonates given acupuncture during heel prick showed significantly shorter crying duration (OR $-65.30,95 \% \mathrm{CI}-101.60$ to -29.00$)$ and lower neonatal infant pain scale scores (OR $-2.00,95 \%$ CI -3.28 to -0.72$)(37)$.

Mumps. One Cochrane review examined the efficacy of acupuncture for mumps and included only one RCT comprising 239 participants. The authors found that the acupuncture group had a higher recovery rate than the control group (RR $1.88,95 \% \mathrm{CI} 1.53-2.30$ ), but took a longer time to recover (MD $0.42,95 \%$ CI $0.31-0.53)(38)$.

Myopia. One Cochrane review assessed acupuncture for myopia and included two RCTs (131 participants) (39). The authors did not perform a meta-analysis and no conclusions can be drawn about the benefit of coacupressure for slowing the progress of myopia in children.

Amblyopia. One non-Cochrane review examined the efficacy of acupuncture for amblyopia, including 14 RCTs (2,662 participants) (40). The authors found that the acupuncture group had higher rates of clinical improvements than the conventional treatment group (RR 1.16, 95\% CI 1.12-1.19).

\section{Safety}

In this overview, only six reviews reported AEs. Three reviews reported specific AEs $(10,25,28)$. Two reviews stated that no AEs were associated with the use of acupuncture $(11,17)$. One review mentioned AEs, but the specific AEs were unclear (27). See Table 5 for the reported AEs of included systematic reviews.

\section{DISCUSSION}

This overview identified 24 systematic reviews, providing us with a comprehensive picture of acupuncture for pediatric conditions. Although most of the included studies could not draw firm conclusions because of the small size of the included trials or their low methodological quality, 


\section{Overview of Acupuncture in Children $\quad$ Systematic Review}

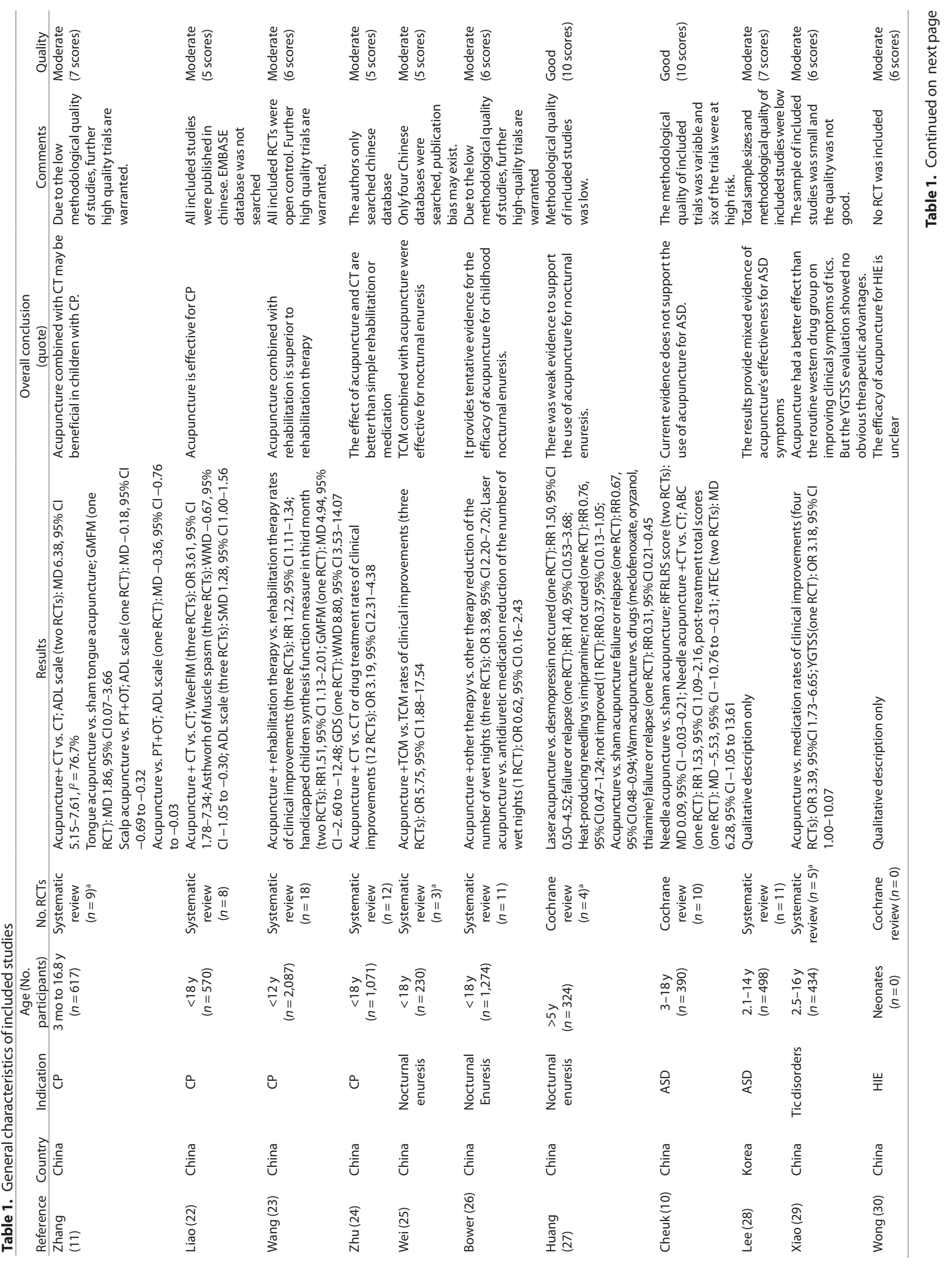




\section{Systematic Review | Yang et al.}

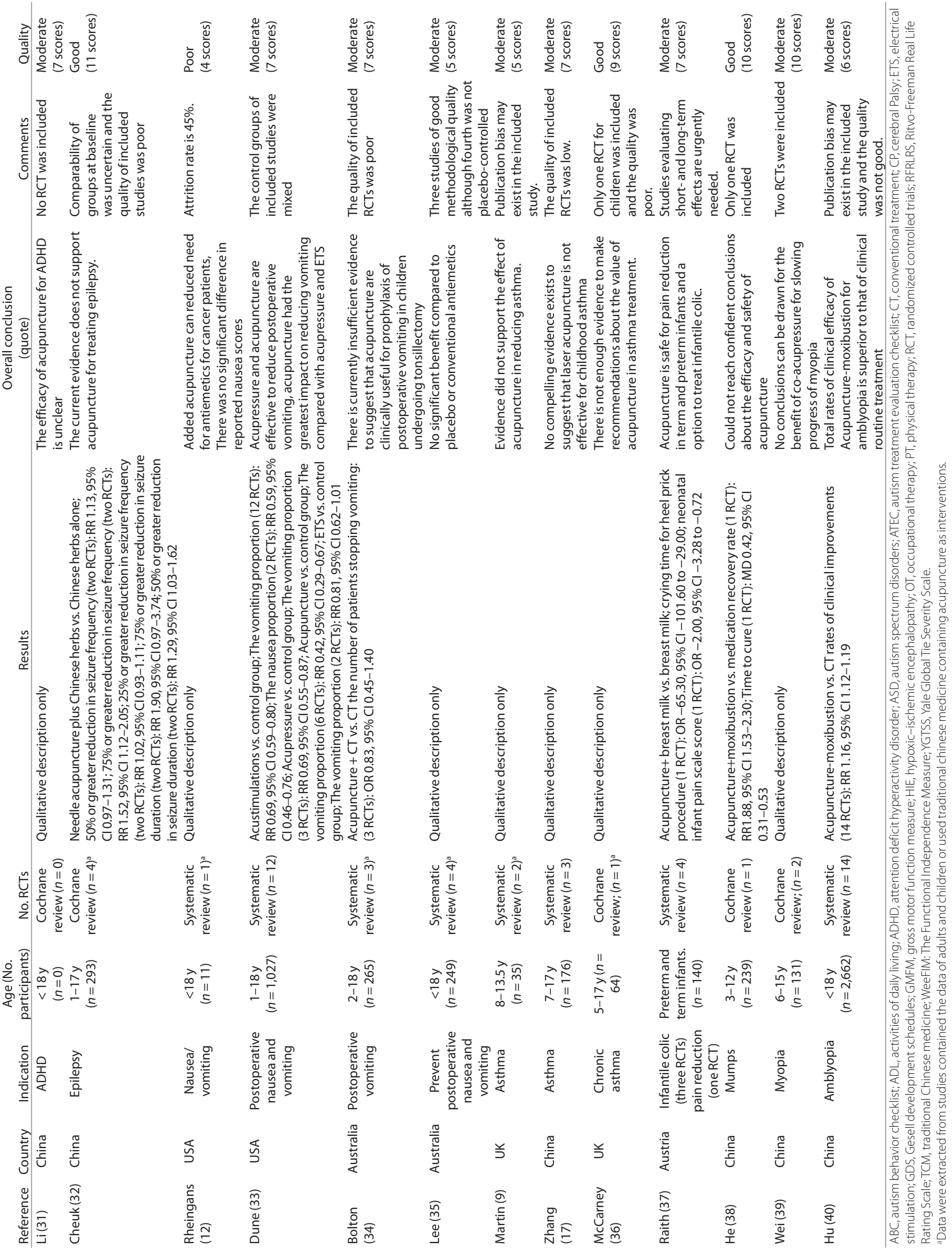


the efficacy of acupuncture for some pediatric conditions is promising, such as for cerebral palsy, nocturnal enuresis, tic disorders, amblyopia, and pain reduction. However, the efficacy of acupuncture for hypoxic-ischemic encephalopathy, attention deficit hyperactivity disorder, mumps, and myopia is unclear and the efficacy for ASD, asthma, and nausea/vomiting is controversial. Current evidence does not support the use of acupuncture for pediatric epilepsy. In this overview, we found that acupuncture was well tolerated and no fatal side effects were reported.

Table 2. The association between different systematic reviews and conclusions

\begin{tabular}{lccc}
\hline Items & $\begin{array}{c}\text { Neutral or } \\
\text { negative results }\end{array}$ & $\begin{array}{c}\text { Positive } \\
\text { results }\end{array}$ & $P$ \\
\hline High-quality systematic reviews & 6 & 0 & 0.052 \\
$\begin{array}{l}\text { Medium or low-quality systematic } \\
\text { reviews }\end{array}$ & 9 & 9 & \\
Cochrane systematic reviews & 8 & 0 & 0.009 \\
Noncochrane systematic reviews & 7 & 9 & \\
\hline
\end{tabular}

In general, the quality of systematic reviews was poor, and only one fifth of reviews were considered to be high quality. The quality of Cochrane systematic reviews was better than that of non-Cochrane systematic reviews. Most systematic reviews did not register the study protocol before conducting the review and did not provide a list of the characteristics of included and excluded studies, with the only exceptions being Cochrane reviews. Only nine out of 24 reviews (37.5\%) of reviews conducted an assessment of publication bias, so we could not judge whether publication bias existed. The status of publication (i.e., gray literature) was used as an inclusion criterion was found in $20.8 \%$ of reviews, so the selecting bias may exist. The potential source of support of the systematic review was clearly acknowledged in $54 \%$ of reviews.

We also found that Cochrane systematic reviews tended to yield less positive results than non-Cochrane systematic reviews, and high-quality systematic reviews tended to yield neutral or negative results. So the poor quality of the systematic reviews makes analysis of the efficacy of acupuncture for children complex.

Table 3. The quality assessment of included systematic reviews

\begin{tabular}{|c|c|c|c|}
\hline AMSTAR question & Yes $(n(\%))$ & Partial or can't tell $(n(\%))$ & No $(n(\%))$ \\
\hline 1. Was an "a priori" design provided? & $8(33.3 \%)$ & 0 & $16(66.7 \%)$ \\
\hline 2. Was there duplicate study selection and data extraction? & 19 (79.2\%) & $5(20.8 \%)$ & 0 \\
\hline 4. Was the status of publication (i.e., gray literature) used as an inclusion criterion? & $5(20.8 \%)$ & 0 & $19(79.2 \%)$ \\
\hline 5. Was a list of studies (included and excluded) provided? & $6(25 \%)$ & $18(75 \%)$ & 0 \\
\hline 7. Was the scientific quality of the included studies assessed and documented? & $24(100 \%)$ & 0 & 0 \\
\hline 8. Was the scientific quality of the included studies used appropriately in formulating conclusions? & $24(100 \%)$ & 0 & 0 \\
\hline 9. Were the methods used to combine the findings of studies appropriate? & $21(87.5 \%)$ & $3(12.5 \%)$ & 0 \\
\hline 10. Was the likelihood of publication bias assessed? & $9(37.5 \%)$ & $2(8.3 \%)$ & $13(54.2 \%)$ \\
\hline 11. Was the conflict of interest stated? & $13(54.2 \%)$ & 0 & $11(45.8 \%)$ \\
\hline
\end{tabular}

Table 4. The subgroup analysis of quality assessment according to type of systematic review

\begin{tabular}{|c|c|c|c|c|c|c|c|}
\hline \multirow[b]{2}{*}{ AMSTAR question } & \multicolumn{3}{|c|}{$\begin{array}{c}\text { Cochrane systematic } \\
\text { reviews }\end{array}$} & \multicolumn{3}{|c|}{$\begin{array}{c}\text { Non-cochrane } \\
\text { systematic reviews }\end{array}$} & \multirow[b]{2}{*}{$P$ value } \\
\hline & Yes & Unclear & No & Yes & Unclear & No & \\
\hline 1. Was an "a priori" design provided? & 8 & 0 & 0 & 0 & 0 & 16 & 0.000 \\
\hline 2. Was there duplicate study selection and data extraction? & 8 & 0 & 0 & 11 & 5 & 0 & 0.130 \\
\hline 3. Was a comprehensive literature search performed? & 8 & 0 & 0 & 10 & 0 & 6 & 0.066 \\
\hline 4. Was the status of publication (i.e. grey literature) used as an inclusion criterion? & 5 & 0 & 3 & 0 & 0 & 16 & 0.001 \\
\hline 5. Was a list of studies (included and excluded) provided? & 6 & 2 & 0 & 0 & 16 & 0 & 0.000 \\
\hline 6. Were the characteristics of the included studies provided? & 6 & 2 & 0 & 16 & 0 & 0 & 0.101 \\
\hline 7. Was the scientific quality of the included studies assessed and documented? & 8 & 0 & 0 & 16 & 0 & 0 & - \\
\hline 8. Was the scientific quality of the included studies used appropriately in formulating conclusions? & 8 & 0 & 0 & 16 & 0 & 0 & - \\
\hline 9. Were the methods used to combine the findings of studies appropriate? & 5 & 3 & 0 & 16 & 0 & 0 & 0.028 \\
\hline 10. Was the likelihood of publication bias assessed? & 4 & 2 & 2 & 5 & 0 & 11 & 0.041 \\
\hline 11. Was the conflict of interest stated? & 8 & 0 & 0 & 5 & 0 & 11 & 0.002 \\
\hline
\end{tabular}


Table 5. The reported AEs in included studies

\begin{tabular}{lll}
\hline References & \multicolumn{1}{c}{ The type of ADR ( $n=$ frequency) } & $\begin{array}{c}\text { Relevance between } \\
\text { AEs and acupuncture }\end{array}$ \\
\hline Lee (28) & $\begin{array}{l}27.3 \%(3 / 11) \text { of included studies mentioned AEs. One RCT reported no adverse events, while two } \\
\text { RCTs reported minor side effects (minor superficial bleeding crying, irritability during treatment, } \\
\text { worsening hyperactivity and ritualistic behavior), the frequency of AEs was unclear }\end{array}$ & Unclear \\
Cheuk (10) & $\begin{array}{l}\text { Worsening of sleeping pattern }(n=1) \text {, superficial bleeding, crying due to fear or pain, irritability, } \\
\text { worsening of hyperactivity and ritualistic behavior, the frequency of other AEs was unclear. }\end{array}$ \\
Wei (25) & $\begin{array}{l}\text { Painful bi-auricular stimulation }(n=3), \text { eczema }(n=2), \text { mild pain, heat or swelling while pressing, the } \\
\text { frequency of other AEs was unclear }\end{array}$ & Unclear \\
Zhang (11) & Reported AEs, but the specific AEs were unclear \\
Zhang (17) & Reported AEs, but the specific AEs were unclear & No relevance \\
Huang (27) & One trial report stated that there were no adverse effects and the remainder did not mention them. & No relevance \\
\hline
\end{tabular}

$\mathrm{AE}$, adverse events; $\mathrm{RCT}$, randomized controlled trials.

As to the safety of acupuncture in children, we found AEs were often ignored or not reported in included reviews. Only six reviews reported the occurrence of AEs, but some important data about the occurrence time, management of AEs, and the definite relationship between AEs and acupuncture was also inadequately reported; future studies should ideally pay more attention to the safety aspect of acupuncture. In general, acupuncture was well tolerated and no fatal side effects were reported, which was in accordance with some reviews evaluating the safety of acupuncture for children. One review included 37 reports and identified a total of 279 AEs, of which 25 AEs were serious, one was moderate, and 253 were mild (incidence $11.8 \%$ 95\% CI: 10.1-13.5) (3). The authors found that many of the serious AEs might have been caused by substandard practice, and that acupuncture is safe when performed by appropriately trained practitioners. Another review included 22 RCTs involving 1,865 participants and reported that the risk of AEs and serious AEs occurring from acupuncture in pediatrics is estimated to be $1.55 / 100$ and 5.36/10,000 treatments respectively (21).

Some limitations of this overview were identified: (i) A quantitative analysis of included reviews could not be conducted owing to statistical and clinical heterogeneity, (ii) The author(s) of included reviews were not contacted to enable judging of whether their methodological quality was assessed appropriately, (iii) Only reviews published in English or Chinese were included. Searches of non-English databases might have yielded further information, but it might be difficult to access local literature internationally as articles might not be indexed in conventional databases and access to local journals might be restricted. However, the priority for highquality research is generally to be published in English journals, with Chinese databases also included in this review as acupuncture originated in China and there are many studies published in Chinese, (iv) Only systematic reviews were included, so some individual RCTs evaluating the efficacy of acupuncture may have been missed.

\section{Conclusion}

The efficacy of acupuncture for some diseases is promising and there have been no fatal side effects reported. Further highquality studies are justified, with five diseases in particular (cerebral palsy, nocturnal enuresis, tic disorders, amblyopia, and pain reduction) as research priorities.

\section{METHODS}

\section{Search Strategy}

Electronic literature searches were carried out in the Cochrane Library (2014, Issue 7), PubMed (1966-2014.7), EMBASE (19742014, Issue 7), Chinese Biomedical Literature Database (CBM, 1978-2014.7), China National Knowledge Infrastructure (19802014.7), Chinese Science and Technique Journals Database (VIP, 1989-2014.7), Wanfang Data (http://www.wanfangdata.com/) (1990-2014.7), and the relevant reference lists. The search terms were "acupuncture", "acupressure", "auricular", "moxibustion", "acupoint", "electric acupuncture", "meridian", "systematic review", "metaanalysis", "pediatrics", "infant", "neonate", "newborn", "child" and "adolescent". The search was restricted to human studies, and the language was restricted to English and Chinese. The search was independently conducted by two investigators and discrepancies were resolved by discussion.

\section{Study Selection}

Systematic reviews and meta-analysis of RCTs or quasi-RCTs evaluating any acupuncture modality (i.e., classical acupuncture, acupressure, moxibustion, scalp acupuncture, body acupuncture, auricular acupuncture, tongue acupuncture, injection acupuncture, electroacupuncture, or any combination of the above) for any type of childhood diseases were included.

We included all reviews including patients under the age of $18 \mathrm{y}$ with any disease regardless of gender, nationality, or outpatient therapy or inpatient therapy.

Reviews were excluded if they: (i) were nonsystematic reviews, (ii) compared the efficacy of different acupuncture therapies, or (iii) included both children and adults and data of children could not be extracted separately.

\section{Data Extraction}

Data were recorded using a standardized data collection form. The data extraction form included: (i) country of the first author, (ii) number of trials included, (iii) indication of included reviews, (iv) patient demographic and clinical data, (v) interventions, (vi) main outcomes and conclusions, and (vii) adverse events (AEs). Data extraction was independently conducted by two investigators and discrepancies were resolved by discussion.

\section{Assessment of Methodological Quality}

Two reviewers independently assessed the methodological quality of all included systematic reviews using The Measurement Tool to Assess Systematic Reviews (AMSTAR). This tool is an 11-item questionnaire requiring assessors to answer "yes", "no", "partial or can't tell". The scores range from 1 to 11 . In our review, a score of 9-11 was considered as high quality, score of $5-8$ as moderate quality, and 
a score of $0-4$ as low quality (41). Discrepancies in their evaluations were discussed and agreed upon through consensus.

\section{Statistical Methods}

A quantitative analysis of included systematic reviews was not conducted owing to statistical and clinical heterogeneity. A narrative summary of included studies has been provided instead.

\section{ACKNOWLEDGMENTS}

C.Y. and Z.H. contributed equally to this study. C.Y.: designed the review, collected data, developed the search strategy, undertook searches, appraised the quality of papers, selected trials for inclusion, extracted data from papers. Data management: carried out analysis and interpretation of the data and wrote the review. Z.H.: collected data, undertook searches, appraised the quality of papers; selected trials for inclusion, extracted data from papers. Data management: checked the data and wrote the review. L.Z.: designed the review, collected data, undertook searches, appraised the quality of papers; selected trials for inclusion, extracted data from papers. Data management: checked the data and commented on drafts for previous version. Q.G.: collected data, undertook searches, appraised the quality of papers; selected trials for inclusion, extracted data from papers.

\section{STATEMENT OF FINANCIAL SUPPORT}

This research was funded by the Natural Science Foundation of China: Evidence based establishment of evaluation index system for pediatric rational use in China. (No drug. 81373381)

Disclosure: The authors declare that they have no financial relationships relevant to this article to disclose. The authors declare that they have no competing interests.

\section{REFERENCES}

1. Barnes PM, Powell-Griner E, McFann K, et al. Complementary and alternative medicine use among adults: United States, 2002. Adv Data 2004;343:1-19.

2. Barnes PM, Bloom B, Nahin RL. Complementary and alternative medicine use among adults and children: United States, 2007. Natl Health Stat Report 2008;10:1-23.

3. Adams D, Cheng F, Jou H, Aung S, Yasui Y, Vohra S. The safety of pediatric acupuncture: a systematic review. Pediatrics 2011;128:e1575-87.

4. Liu F, Li ZM, Jiang YJ, Chen LD. A meta-analysis of acupuncture use in the treatment of cognitive impairment after stroke. J Altern Complement Med 2014;20:535-544.

5. Sniezek DP, Siddiqui IJ. Acupuncture for treating anxiety and depression in women: a clinical systematic review. Med Acupunct 2013;25:164-72.

6. Chao GQ, Zhang S. Effectiveness of acupuncture to treat irritable bowel syndrome: a meta-analysis. World J Gastroenterol 2014;20:1871-7.

7. Lam M, Galvin R, Curry P. Effectiveness of acupuncture for nonspecific chronic low back pain: a systematic review and meta-analysis. Spine (Phila Pa 1976) 2013;38:2124-38.

8. Lin YC, Lee AC, Kemper KJ, Berde CB. Use of complementary and alternative medicine in pediatric pain management service: a survey. Pain Med 2005;6:452-8.

9. Martin J, Donaldson AN, Villarroel R, Parmar MK, Ernst E, Higginson IJ. Efficacy of acupuncture in asthma: systematic review and meta-analysis of published data from 11 randomised controlled trials. Eur Respir J 2002;20: 846-52.

10. Cheuk DK, Wong V, Chen WX. Acupuncture for autism spectrum disorders (ASD). Cochrane Database Syst Rev 2013;7:1-92.

11. Zhang Y, Liu J, Wang J, He Q. Traditional Chinese Medicine for treatment of cerebral palsy in children: a systematic review of randomized clinical trials. J Altern Complement Med 2010;16:375-95.

12. Rheingans JI. A systematic review of nonpharmacologic adjunctive therapies for symptom management in children with cancer. J Pediatr Oncol Nurs 2007;24:81-94.

13. Zhang S, Li N, Liu M. Use of acupuncture for stroke in China. Acupunct Med 2009;27:146.

14. Cabýoglu MT, Ergene N, Tan U. The mechanism of acupuncture and clinical applications. Int J Neurosci 2006;116:115-25.

15. Ernst E. Acupuncture-a critical analysis. J Intern Med 2006;259:125-37.
16. Zhang JH, Wang D, Liu M. Overview of systematic reviews and meta-analyses of acupuncture for stroke. Neuroepidemiology 2014;42:50-8.

17. Whittaker P. Laser acupuncture: past, present, and future. Lasers Med Sci 2004;19:69-80.

18. Xu J, MacKenzi IZ. The current use of acupuncture during pregnancy and childbirth. Curr Opin Obstet Gynecol 2012;24:65-71.

19. Zhang J, Li X, Xu J, Ernst E. Laser acupuncture for the treatment of asthma in children: a systematic review of randomized controlled trials. J Asthma 2012;49:773-7.

20. Hunt K, Ernst E. The evidence-base for complementary medicine in children: a critical overview of systematic reviews. Arch Dis Child 2011;96:769-76.

21. Jindal V, Ge A, Mansky PJ. Safety and efficacy of acupuncture in children: a review of the evidence. J Pediatr Hematol Oncol 2008;30:431-42.

22. Liao C, Zhou JB. Meta-analysis on acupuncture in treatment of cerebral palsy. Chinese General Practice 2011;14:1229-31.

23. Xu W, Du YH, Jun X, Zhang YY. Acupuncture versus rehabilitation therapy for cerebral palsy: a systematic review. Liaoning J Traditional Chinese Med 2011;38:408-413.

24. Jing Z, Zhang LY, Shao XN. Meta analysis of acupuncture treatment for children with cerebral palsy. Guiding J Traditional Chinese Medicine and Pharmacy 2013;19:62-65.

25. Wei SZ, Zheng FJ. A Meta analysis of acupuncture combined with traditional Chinese medicine in the treatment of nocturnal enuresis in children. Chinese J Healthy Birth \& Child Care 2013;19:78-81.

26. Bower WF, Diao M, Tang JL, Yeung CK. Acupuncture for nocturnal enuresis in children: a systematic review and exploration of rationale. Neurourol Urodyn 2005;24:267-72.

27. Huang T, Shu X, Huang YS, Cheuk DK. Complementary and miscellaneous interventions for nocturnal enuresis in children. Cochrane Database Syst Rev 2011;12:1-68.

28. Lee MS, Choi TY, Shin BC, Ernst E. Acupuncture for children with autism spectrum disorders: a systematic review of randomized clinical trials. J Autism Dev Disord 2012;42:1671-83.

29. Xiao L, Chen YW, Du YH, Gao X, Lin XM, Sun P. Evaluation of clinical randomized control trials of acupuncture for treatment of multiple tics disorders. Lishizhen Med Materia Medica Res 2010;21:1199-1202.

30. Wong V, Cheuk DK, Chu V. Acupuncture for hypoxic ischemic encephalopathy in neonates. Cochrane Database Syst Rev 2013;1:CD007968.

31. Li S, Yu B, Zhou D, He C, Kang L, Wang X, Jiang S, Chen X. Acupuncture for attention deficit hyperactivity disorder (ADHD) in children and adolescents. Cochrane Database Syst Rev 2011;4:1-31.

32. Cheuk DK, Wong V. Acupuncture for epilepsy. Cochrane Database Syst Rev 2008;8:1-103.

33. Dune LS, Shiao SY. Metaanalysis of acustimulation effects on postoperative nausea and vomiting in children. Explore (NY) 2006;2:314-20.

34. Bolton CM, Myles PS, Nolan T, Sterne JA. Prophylaxis of postoperative vomiting in children undergoing tonsillectomy: a systematic review and meta-analysis. Br J Anaesth 2006;97:593-604.

35. Lee A, Done ML. The use of nonpharmacologic techniques to prevent postoperative nausea and vomiting: a meta-analysis. Anesth Analg 1999;88:1362-9.

36. McCarney RW, Brinkhaus B, Lasserson TJ, Linde K. Acupuncture for chronic asthma. Cochrane Database Syst Rev 2009;3:1-51.

37. Raith W, Urlesberger B, Schmölzer GM. Efficacy and safety of acupuncture in preterm and term infants. Evid Based Complement Alternat Med 2013;2013:739414.

38. He J, Zheng M, Zhang M, Jiang H. Acupuncture for mumps in children. Cochrane Database Syst Rev 2012;9:CD008400.

39. Wei ML, Liu JP, Li N, Liu M. Acupuncture for slowing the progression of myopia in children and adolescents. Cochrane Database Syst Rev 2011;9:1-31.

40. Hu YH, Li Y, Han DY, Wang XC, Wang FC, Wang ZH. Meta analysis on acupuncture-moxibustion for amblyopia. Chinese Acupuncture \& Moxibustion 2014;34:1-4.

41. Shea BJ, Hamel C, Wells GA, et al. AMSTAR is a reliable and valid measurement tool to assess the methodological quality of systematic reviews. J Clin Epidemiol 2009;62:1013-20. 